\title{
Influence of Subliminal Cueing on Visual Search Tasks
}

Bastian Pfleging,

Niels Henze,

Albrecht Schmidt

VIS, University of Stuttgart VIS, University of Stuttgart

70569 Stuttgart, Germany

\{first.last\}@vis.uni-stuttgart.de $\{$ raude, reitschbn\}@studi.

informatik.uni-stuttgart.de

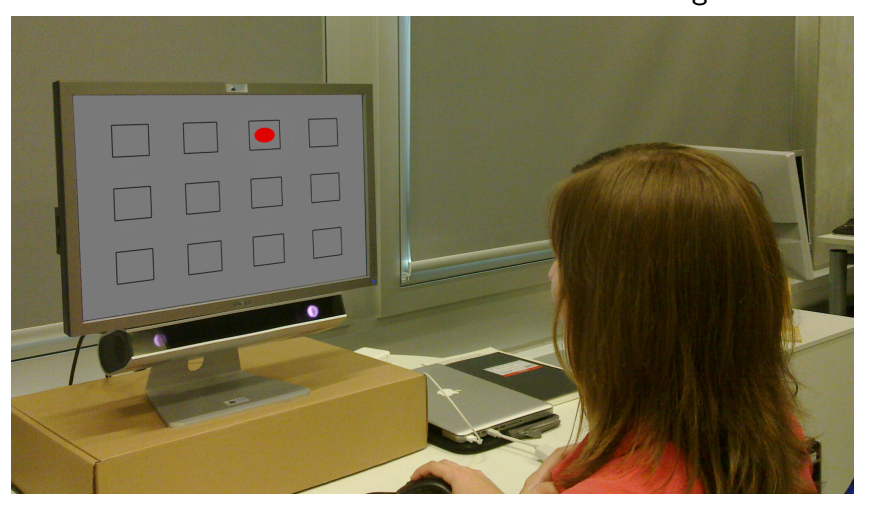

Figure 1: A participant follows a red ellipse with her eyes. This target is moving within the matrix of rectangles. Cues of

different intensities are given for the next location of the target to find out how they influence the visual behavior of the user.

Copyright is held by the author/owner(s).

CHI 2013 Extended Abstracts, April 27-May 2, 2013, Paris,

France.

ACM 978-1-4503-1952-2/13/04.

\begin{abstract}
The phenomenon of subliminal perception is studied in psychology and is a compelling idea to unobtrusively yet effectively convey information from the computer to the user. Previous research reports conflicting results regarding the effectiveness of subliminal stimuli in graphical user interfaces (GUIs). These experiments are often reported on application level and are generally hard to reproduce. We aim at isolating the effect to learn how subliminal cueing can become a basis for future GUI widgets. Therefore, we look at specific properties and functions that can be realized using subliminal presentation. In this paper we present our ongoing work towards highlighting parts of the interface to guide the user's gaze. In the conducted study, participants had to look at targets appearing at different screen locations. Using different cueing variants, the participants received hints of the next location. The results indicate that visible cues are effective, whereas the studied simple and non-blinking subliminal cueing method does not improve visual search performance.
\end{abstract}

\section{Author Keywords}

Graphical user interface; subliminal cues; user guidance

\section{ACM Classification Keywords}

H.5.2. [Information Interfaces and Presentation (e.g. $\mathrm{HCl}$ )]: User Interfaces 


\section{Introduction}

Subliminal information perception and communication has been researched in psychology and the effect is well described. Besides the scientifically grounded knowledge, there are many myths surrounding this concept, especially its potential application in advertising [11]. Researchers in human-computer interaction have made attempts to create meaningful applications using subliminal information presentation $[1,9,10]$. However, many of them are described at a general level with many factors that impacted these experiments. Similarly, there are researchers who argue that subliminal interfaces do not work [2].

We aim at systematically analyzing the opportunities for subliminal cues and their (assistive) potential for GUI widgets. An example of a potential use case is: If a user writes an e-mail and mentions an 'attachment' but did not attach any file yet, current e-mail clients add for instance a colored bar above the e-mail body stating that the user might have forgotten to attach a file. Adding subliminal effects to GUI widgets, the idea is to implicitly direct the user's gaze to the attachment button as a reminder. This is attractive as it could be realized without adding visual content. Also, the concept could be used to implement notifications such as in calendars or task bars. Compared to colored bars, we think that subliminal cues can help to make the user feel empowered. Cues similar to the ones we investigated could add a dimension to the design space of GUls. From a practical perspective they have the potential to scale up to more hints on a limited screen space.

In the following we assess the effectiveness of a particular subliminal cueing method and compare it to no cues and visual cues. The expectation is that the subliminal cues reduce the time required to complete visual search tasks. We first discuss the terminology and related work. Next we describe our experiment and the results. We conclude that the presented type of subliminal cues does not provide a means for improving the performance in visual search tasks.

\section{Terminology and Related Work}

Since the late 18th century, scientists have experimented with subliminal stimuli. Besides critical concerns, the idea to influence a user's decision, behavior, and habits or to support one's memory this way is exciting.

Terminology Our research focuses on the continuum between subliminal, supraliminal, and clearly visible stimuli. As the terminology of subliminal stimuli is not consistent in literature, we first want to establish a terminology which we will use throughout the paper. While there are other potential modalities, our focus is on visual stimuli. Similar to the definition by Chalfoun and Frasson [3], we define a stimulus as being subliminal when a user cannot consciously report its existence (e.g., when it appears for a short time only). In cognitive neuroscience, the term of conscious perception or processing is well established and defined. One way to distinguish between subconscious (unconscious) and conscious perception is a temporal threshold [5]. In our case, we look at visual (color) modulations of the interface which may even be shown longer than the afore mentioned temporal threshold but which are (almost) incognizable due to their slightness.

Subliminal Cueing to Provide Help and Support Different projects have experimented with subliminal communication to assist users of interactive systems. Several projects produced practical applications that create a positive effect for the user. Providing help to the user with subliminal cues seems promising if three conditions are met [8]: (1) messages must support the user, 


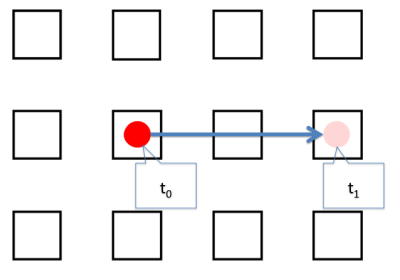

Figure 2: Study procedure: After a target has been shown for two seconds $\left(t_{0}\right)$, a subliminal or visible cue for the next target location is given for one second $\left(t_{1}\right)$. Afterwards, the target appears for two seconds at this location. During the two baseline conditions, neither cue nor target are shown during $t_{1}$
(2) a message must be timely at a tricky stage of work, and (3) the user's task must be cognitively demanding.

An early study in the human-computer interaction domain has been presented by Wallace et al. [13]. They used subliminal cues for a task-oriented support within a text editor by showing shortcuts. The experiment showed that subliminal cues had a positive effect during demanding, confusing, or difficult tasks. Receiving subliminal cues resulted in asking for help less frequently. DeVaul et al. [6] conducted an experiment where participants first had to memorize several name-face pairs. Next, these faces were presented to the participants. When the participants were wearing a head-mounted display showing subliminally a person's name, the ability to recall the correct name could be increased - even when offering a misleading cue. For a computerized quiz, Chalfoun and Frasson used subliminal messages to support learning solution strategies. Using subliminal messages resulted in $44 \%$ less errors $[3,4]$.

Directing the User's Gaze Another research direction tries to direct the gaze instead of transmitting information. Bailey et al. coined the term "Subtle Gaze Direction" (SGD) for a technique to direct a user's gaze [1]. This technique exploits the poor acuity of the human visual system: While the visual acuity at the fovea (foveal vision) is very high, it rapidly decreases towards the peripheral region. In contrast, the peripheral vision can react faster to stimuli than the foveal vision. Therefore, SGD modulates a representation in the peripheral region of the user's view to trigger peripheral vision and to draw the foveal vision to this area of interest (AOI). The phenomenon of saccadic masking [7] is simultaneously used to hide the modulation: The effect is removed once a saccadic movement towards the $\mathrm{AOI}$ is detected and before the foveal vision can fixate at the AOI. Peripheral vision was triggered by changing luminance and color temperature at a rate of $10 \mathrm{~Hz}$. Thus, they successfully attracted the viewer's foveal vision and moved away the viewers' focus points from salient areas to less interesting parts of an image.

McNamara et al. researched the effect of luminance modulation on search task performance in digital images. Using SDG they compared the effects of obvious, subtle, and no highlights [9] on the users' performance by letting them count transparent "bubbles" in a digital image. The results show similar precision gains using subtle and clearly visible cues. In a second trial [10], McNamara et al. added distractors to the images. The distractors did not reduce the performance using subtle highlighting but even increased the accuracy due to breaking the usual attention people give to sharp areas, edges, and faces. However, no significant difference was found between the subtle and obvious presentation.

Sridharan et al. [12] investigated if SGD can improve the efficiency of digital mammography training. An expert radiologist prepared training images by annotating a typical gaze path that experts would use to check such images for abnormalities. During a training session as well as during a short-term follow-up study the participants that received SGD treatments performed significantly better than the static control group. However, for a long-term follow-up session, no significant effect was found.

\section{Experiment}

While alternating modulations are used for SGD, we are interested in non-blinking cues and explore the continuum for cues that can be used for notifications and information presentation ranging from invisible to subliminal and clearly visible cues. Therefore, we conducted a repeated measures experiment to compare six different user-calibrated cueing 


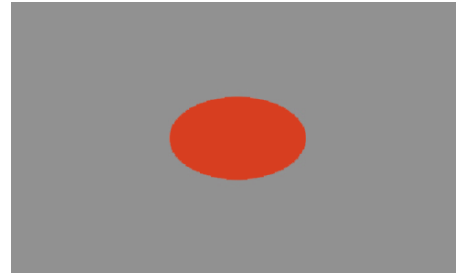

Figure 3: Intensity calibration for foveal vision: To detect the fovea visibility threshold, the participan looks at the center of the screen where a red ellipse will fade in $\mathrm{S} /$ he shall press a key once noticing a change.

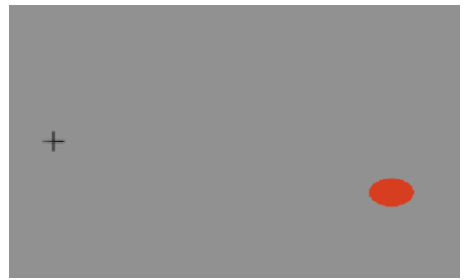

Figure 4: Intensity calibration for peripheral vision: Similar to the foveal calibration, the participant looks at a crosshair while an ellipse fades in at the other side of the screen. A key press determines the visibility threshold value once $\mathrm{s} /$ he notices a change. intensities with two control conditions (no cue) in a search task. The participants' assignment was to visually follow a red ellipse (target) shown at different discrete locations as fast as possible. A cue is presented at the next target location to provide a hint to the participant.

\section{Method}

The independent variable is the cueing intensity with seven levels (see Table 1 ) that is calibrated per participant: Besides a baseline as control condition (no cue), two subliminal levels, and one obviously visible level are used. Each level is tested first with a fixed intensity and next with a distance adaptive intensity (gaze position $\leftrightarrow$ target) to compensate different visibility thresholds for foveal and peripheral vision. For each condition, a red ellipse as target randomly skips 20 times between 12 fixed boxes on the screen: It is shown for $2 \mathrm{~s}$ in one box. Next, it disappears and a non-blinking cue (like a "ghost image") is given for $1 \mathrm{~s}$ at the next location. Finally, the target becomes completely visible for $2 \mathrm{~s}$ at this location (Figure 2 ) and so on. We use a fixed order of conditions: In order to not have the users actively looking for cues, we do not mention them before and start with a control condition (no cue shown between visible periods). Then, we increase the visibility of the cue with each (non-randomized!) condition until full visibility and terminate with another control condition (see Table 1). The dependent variable is the time difference between occurrence of a target and the the subject's first fixation on this AOI.

Participants 18 participants ( 5 female) between 17 and 29 years $(M=23, S D=3.5)$ took part in our study. We recruited them through our university's mailing lists and compensated them. All participants had normal or corrected to normal vision. We ensured that they had no prior knowledge of the purpose and goal of the study.
Apparatus All participants execute the task under the same lighting conditions with only artificial light. We use a calibrated DELL P2210f 22 " screen with a refresh rate of $60 \mathrm{~Hz}$ and a resolution of $1680 \mathrm{px} \times 1050 \mathrm{px}$. An SMI RED250 remote eye tracking system tracks the user gaze at $250 \mathrm{~Hz}$. The eye tracker is attached to the screen as shown in Figure 1.

The user interface is rendered in full-screen mode with OpenGL and the total delay of processing and preparing the stimulus is about $8 \mathrm{~ms}$. The 12 boxes are drawn as black frames $(185 \mathrm{px} \times 150 \mathrm{px})$ on a grey background (see Figures 1 and 2). Each user is seated about $70 \mathrm{~cm}$ from the screen and is instructed not to move. The boxes are uniformly distributed across the screen. A red ellipse $(r, g, b)=(255,0,0)$ is used as stimulus at a size of $4.27^{\circ} \times 3.47^{\circ}$. In the subliminal cases, the same ellipse is used, but its fixed translucency $\alpha_{f}$ or its dynamic translucency $\alpha_{d}$ is modified, also considering the user-calibrated visibility thresholds. To adapt the translucency to the current distance between gaze position and target location during distance-adaptive conditions, the following term is used to calculate the dynamic translucency $\alpha_{d}$

$$
\alpha_{d}=\alpha_{\text {foveal }}+\frac{d}{d_{\max }} *\left(\alpha_{\text {peripheral }}-\alpha_{\text {foveal }}\right)
$$

$\alpha_{\text {foveal }}$ represents the current user-calibrated value used for translucency for foveal vision and $\alpha_{\text {peripheral }}$ for peripheral vision respectively. The Euclidean distance $d$ between the center of the target center and gaze position is updated with $250 \mathrm{~Hz}$. The display's diagonal of $1972 \mathrm{px}$ is used as the maximal distance $d_{\max }$.

Procedure After welcoming a participant we explain the procedure of the study without describing the specific goal 


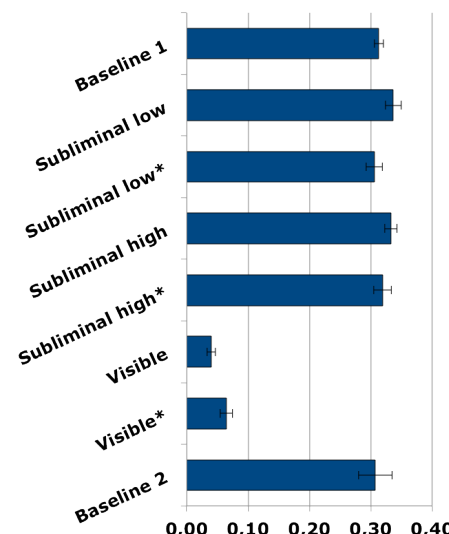

$0,00 \quad 0,10 \quad 0,20 \quad 0,30 \quad 0,40$

Time [s]

Figure 5: Average gaze arrival time (error bars show standard error).

\begin{tabular}{|l|r|r|}
\hline Condition & M[ms] & SD \\
\hline baseline 1 & 313 & 32 \\
\hline subliminal low & 337 & 55 \\
\hline subliminal low* & 306 & 55 \\
\hline subliminal high & 333 & 41 \\
\hline subliminal high* & 319 & 60 \\
\hline visible & 40 & 29 \\
\hline visible* & 64 & 43 \\
\hline baseline 2 & 307 & 116 \\
\hline
\end{tabular}

Table 1: Order and average gaze arrival times of the eight conditions. Distance-adaptive conditions are marked by ${ }^{*}$, all other conditions had static translucency values. of the experiment. Before starting the experiment, the eye tracker is calibrated (5-point method). The participants are told to keep their head as steady as possible during the experiment (the eye tracker is able to correct small head movement, we do not use a chin rest)

As a first part of our study (introduced as "reaction test"), we measure the minimal color difference (visibility threshold) a participant is able to detect in two steps. We first measured the threshold for foveal vision (see Figure 3): A filled red ellipse with slightly varying radius is faded in at the center of the screen. The ellipse is completely translucent at first and we slowly increase $(0.03 \%$ every $0.3 \mathrm{~s}$ ) its visibility. We ask the participants to press a button on a keyboard as soon as they spot a change on the screen. The current translucency threshold is determined

$\mathrm{t}$ when the participant hits the key. This procedure is repeated five times and the mean translucency $t_{\text {foveal }}$ is then used as the threshold for conscious visual perception. Afterwards, we measure this threshold for peripheral vision (see Figure 4). We ask the participants to focus on a crosshair on the left side of the screen. Similarly to the first test, a filled ellipse (slightly varying radius) fades in on the right side of the screen. Again, we calculate the mean translucency value $t_{\text {peripheral }}$ of five consecutive measurements, while ensuring (via eye tracker) that the participants focused the cross line.

\section{All conditions are then tested sequentially. For each} condition, the participant is asked to follow the red ellipse as fast as possible as it randomly skips between the boxes for 20 times as described before. Breaks were given after completion of each condition. During the break we ask the participants if they "recognized something peculiar" without giving a hint for what to look for. After beginning with no cue, the second condition used a fixed translucency of $\alpha_{f}=t_{\text {foveal }} / 2$ for the cue to stay below the participant's personal visibility threshold. Next, cueing was done using the same translucency, but adapting $\alpha_{d}$ it to the distance as described above $\left(\alpha_{\text {foveal }}=t_{\text {foveal }} / 2\right.$,

$\left.\alpha_{\text {peripheral }}=t_{\text {peripheral }} / 2\right)$. After repeating these two steps with $\alpha_{f}=\alpha_{\text {foveal }}=t_{\text {foveal }}$ and $\alpha_{\text {peripheral }}=t_{\text {peripheral }}$, we continued with a clearly visible hint $\left(\alpha_{f}=\alpha_{\text {foveal }}=4 \%\right.$ and $\alpha_{\text {peripheral }}=6 \%$ ) also with and without accounting distance. Finally, we repeated the procedure again without a cue to check for learning effects.

\section{Results}

In our reaction test, we found that most of our participants had a similar visibility threshold for $t_{\text {foveal }}(M=0.7 \%$,

$S D=0.01 \%$ ) while the value $t_{\text {peripheral }}$ for peripheral vision was more diverse $(M=1.7 \%, S D=0.31 \%)$.

The average gaze arrival times are shown in Figure 5 and Table 1. Mauchly's test indicate that the assumption of sphericity had been violated, $\chi^{2}(27)=70.016, p<.001$. A Greenhouse-Geisser corrected ANOVA shows that the time of gaze arrival is affected by the type of cueing,

$F(2.38,40.38)=82.27, p<.001$. A Bonferroni post-hoc test shows that the two clearly visible cues lead to significantly shorter gaze arrival times compared to all other conditions $(p<.001)$. No other significant effect was found.

One participant noticed the cue once during the 4th condition ('subliminal high') and another four participants noticed a cue (1-3 times during the condition) in the least subliminal condition (adaptive 'subliminal high*'), passing the visibility threshold in those conditions. This happened when the ellipse appeared in the same box as before. Nobody noticed a cue during the first three conditions. Two participants reported the subliminal conditions being easier, otherwise only repetitions or other patterns (like position distribution) were reported as peculiar. 


\section{Conclusion}

We conclude that priming with the presented simple, non-blinking subliminal cues is not effective. Our experiment indicates that clearly visible cues significantly improve participants' performance. Using personalized subliminal cues, we could, however, not show that these improve the users' performance - not even compared to the control condition. In contrast to Bailey's findings [1], where subtle cues resulted in an effect, we could neither influence gaze behavior nor increase visual search performance. Despite reports of 'feeling easier' (by 2 participants), measured data opposes any temporal benefits.

As we only measured the eye gaze behavior and the time to visually reach a target, we did not yet collect any further dates. For a future version, we could think of additional measures to uncover other effects like for instances changes in mental task load. Our stimuli were presented on a monochrome background similar to many modern user interfaces but in contrast to experiments like [1]. As the concept of subliminal cueing still seems to be promising, we intend to continue research in this domain, e.g., by looking at more complex subliminal cues. This way, we hope to find benefits for human-computer interaction by using unobtrusive techniques to support graphical user interfaces.

\section{Acknowledgments}

This project was funded from the German Research

Foundation within the Cluster of Excellence in Simulation

Technology (EXC 310/1) at the University of Stuttgart.

\section{References}

[1] Bailey, R., McNamara, A., Sudarsanam, N., and Grimm, C. Subtle Gaze Direction. ACM Trans. Graph. 28, 4 (Sept. 2009), 100:1-100:14.

[2] Campbell, T. The effect of subliminal learning: Using a direct manipulation computer interface. UNF
Theses and Dissertations (Jan. 1993).

[3] Chalfoun, P., and Frasson, C. Subliminal Priming

Enhances Learning in a Distant Virtual 3D Intelligent Tutoring System. IEEE MEEM 3 (2008), 125130.

[4] Chalfoun, P., and Frasson, C. Subliminal Cues While Teaching: $\mathrm{HCl}$ Technique for Enhanced Learning. Advances in Human-Computer Interaction 2011 (2011).

[5] Del Cul, A., Baillet, S., and Dehaene, S. Brain dynamics underlying the nonlinear threshold for access to consciousness. PLoS Biol 5, 10 (Sept. 2007), e260.

[6] DeVaul, R. W., Pentland, A. S., and Corey, V. R. The memory glasses: Subliminal vs. overt memory support with imperfect information. ISWC '03 (2003), 146-153.

[7] Dodge, R. Visual perception during eye movement. Psychological Review 7 (1900), 454-465.

[8] Kelly, R. Subliminal Computing: The Support You Don't See. XRDS 18, 1 (2011), 2528.

[9] McNamara, A., Bailey, R., and Grimm, C. Improving search task performance using subtle gaze direction. In In Proc. APGV 2008 (2008), 5156.

[10] McNamara, A., Bailey, R., and Grimm, C. Search task performance using subtle gaze direction with the presence of distractions. ACM Trans. Appl. Percept. 6, 3 (Sept. 2009), Article 17, 1-19.

[11] Moore, T. Subliminal advertising: What you see is what you get. The Journal of Marketing (1982), 3847.

[12] Sridharan, S., Bailey, R., McNamara, A., and Grimm, C. Subtle Gaze Manipulation for Improved Mammography Training. APGV '11, ACM (2011), 112-112.

[13] Wallace, F., Flanery, J., and Knezek, G. A. The Effect of Subliminal HELP Presentations on Learning a Text Editor. Information Processing \& Management 27, 23 (1991), 211-218. 